\title{
Potential Increased performance of an array of Cherenkov telescopes by means of their positioning using many-objective evolutionary optimizations
}

\author{
Bruno F. Souto ${ }^{a 1}$ and Ulisses Barres de Almeida ${ }^{a 2}$ \\ ${ }^{a}$ Brazilian Center for Research in Physics, Rio de Janeiro, RJ, Brazil
}

Received on Oct 12, 2018 / Accepted on Dec 22, 2018

\begin{abstract}
This paper is concerned with the performance optimization of an array of at most six imaging atmospheric Cherenkov telescopes as a function of their positions on the ground. Two types of telescopes were used, with ranges of detection equal to $300 \mathrm{~m}$ or $500 \mathrm{~m}$. The ideas presented here were developed around an alternative way that employs a modelling step and the implementation of an evolutionary algorithm. We look for configurations that were not investigated by Monte Carlo simulations yet. We found solutions represented by geometric shapes with 3 to 6 telescopes. This is an initial work and the methods developed here have potential applications in other optimization issues on Gamma Ray Astronomy.
\end{abstract}

Keywords: Gamma-ray astronomy, imaging atmospheric Cherenkov telescopes, multi-objective optimization, EliteNSGA-III.

\section{Introduction}

The study of the gamma rays that arrive at Earth allows us to obtain information about its sources in the Universe. When they reach the Earth's atmosphere, gamma rays can give rise to a cascade of particles that propagates toward the ground in a phenomenon known as atmospheric shower. By detecting some particles of this shower, the energy and the arrival direction of the primary gamma ray can be reconstructed. In this way, in the 1980's the first gamma ray source was discovered by the Whipple Telescope when collecting the Cherenkov radiation emitted by the showers [1]. Since then, well over a hundred other sources have been discovered through the use of arrays of Imaging Atmospheric Cherenkov Telescopes (IACTs)[2].

Optimizing the performance of these arrays means increasing the rate of event detection and improving the reconstruction of the primary gamma ray. Currently, experiments in Gamma Ray Astronomy (GRA) contain arrays with at most 5 telescopes [3]. Thus their optimization has been figured out by finding via Monte

\footnotetext{
${ }^{1}$ E-mail Corresponding Author: bsouto@cbpf.br

${ }^{2}$ E-mail: ubarres@cbpf.br
} 
Carlo simulations the best spacing among them in regular and symmetrical geometries and by optimizing the individual parameters of the telescopes (e.g. [4, 5]). The spacing among the telescopes represents a trade-off between a good stereoscopic view of the showers and the requirement of a large effective area for good sensitivity.

On the other hand, still under development, the Cherenkov Telescope Array (CTA) will be an array of almost 100 telescopes, distributed on two sites in both hemispheres [6]. Consequently, CTA optimization demands more complex studies and also a higher computational effort from the Monte Carlo simulations involved. These simulations are usually done with state-of-the-art softwares such as CORSIKA [7] and simtelarray [8, 9], which take into account the showers development, with the hadronic shower acting as a noise in the detection process, the atmospheric model and all the process of the shower detection by the telescope mirrors and its electronic system.

An additional difficulty lies in the fact that the CTA will be a hybrid array, consisting of telescopes of different types, which respond differently to incoming showers of different energies. Previously, for designs of large arrays of homogeneous telescopes, one strategy to circumvent the complexity of large Monte Carlos simulations was the concept of "array cells". A "cell" is a sub-array composed of few telescopes, which can be easily optimised in itself - for example, a square configuration with the four telescopes occupying its vertices. Analyzing the performance of a cell, the performance of a regular arrangement can be estimated by the superposition of $N$ cells $[10,11]$. However, this solution is restricted to symmetric geometries, and is not guaranteed or demonstrated to be the optimal solution for large arrays. In fact, the only tested configurations tend to be the symmetric ones, because of the computational cost of testing a large number of arbitrary configurations.

The strategy proposed in this paper consists in translating into a heuristic model how the optimization objectives depend on the geometry of the telescope array and programming an algorithm that searches for solutions that approximate the optimal configuration sought. The existence of conflicting objectives motivates the use of Evolutionary Optimization techniques. The idea is to create an efficient and easy-to-implement algorithm that generates good solutions for the positioning of telescopes in the array. The proposal of our algorithm is not to replace the Monte Carlo simulations, but to help them. Alternatively it could be used to refine or correct symmetric results. The advantage of the heuristic model is to generate lower computational cost during each algorithm iteration. In another context, on arrays of radio antennas it was found that non intuitive geometries are better than the regular grid and this fact also motivates our search [12].

The paper is organized as follows: Section 2 introduces multi-objective algo- 
rithms and motivates our choice by evolutionary methods. Section 3 states the problem of interest. Section 4 develops the algorithm used for optimization. Section 5 presents the main results and the conclusions are summarized in Section 6.

\section{Multi-objective Optimization and Evolutionary Algorithms}

Evolutionary Algorithms are suitable approaches for solving multi-objective problems. They are based on the manipulation of a set of candidate solutions in order to obtain a progressive improvement of them as the iterations of the algorithm are running, in analogy with the generations of living beings [13]. Some examples are the MOGA, SPEA and NSGA2 algorithms, among others, that are distinguished mainly by the way they classify the solutions, guarantee the survival of the best and preserve the diversity of solutions throughout the search [14].

The desirable characteristics of the evolutionary methods that justified our choice to optimize our specific problem are:

- Possibility of concurrently manipulating a set of solutions at each iteration, because the problem to be solved is multi-objective;

- Presence of strategies to encourage solutions diversity, to achieve convergence of the algorithm and to maintain good processing speed;

- Non-dependence of hypotheses on the search space;

- Flexibility to address possible changes in problem formulation;

- Abscen of an exact algorithm to solve the problem directly.

When dealing with multi-objective optimization problems, the objective functions can be conflicting with each other, i.e., there may not be a single solution that optimizes all of them simultaneously. In this regard, one must use another notion for the optimal solution of the problem.

In this work, an optimal solution means that there is no other solution that improves the value of one goal without worsening the value of another one. Strictly speaking, the optimal solutions are those that belong to the Pareto front [13].

In fact, there may be an infinite number of Pareto-optimal solutions. If additional information on the relative importance among objectives is unknown, all Pareto-optimal solutions are equally important and it can not be said that some solution is better than another. In practice, an evolutionary algorithm searches a finite set of solutions that is as close to the Pareto front as possible. Besides, this kind of algorithm aims to the diversity of the solutions and to perform the optimization with high computational efficiency. Thus, the general purpose of the algorithm is to find the trade-off among the objective functions instead of an optimal solution 
for each objective separately or a single solution, as in the case of global optimization. After having an overview of all other solutions obtained by the algorithm, it is assigned to the Decision Maker to pick one of them [15].

\section{Problem Formulation}

The general formulation of a multi-objective optimization problem can be written as

$$
\begin{array}{rlrl}
\operatorname{minf}_{i}(\mathbf{v}) & i & =1, \ldots, M \\
g_{j}(\mathbf{v}) \geq 0 & j & =1, \ldots, N_{g} \\
h_{k}(\mathbf{v})=0 & k & =1, \ldots, N_{h} \\
v_{n}^{L} \leq v_{i} \leq v_{n}^{H} & n & =1, \ldots, N_{v}
\end{array}
$$

where $g_{j}$ and $h_{k}$ are constraints of the problem, and $\mathbf{v}$ is a vector subject to $N_{v}$ constraints in the variable space.

In our particular case, i.e., the performance optimization of an array of IACTs through the positioning of its $N$ telescopes, there are no problem constraints, thus we neglect the functions $g_{j}$ and $h_{k}$. Our telescope array is arranged in a square flat region of dimensions $2200 \mathrm{~m} \times 2200 \mathrm{~m}$. Due to the finite distance of the telescope's detection, there are some constraints in the variable space to consider a uniform edge around the whole region. Thus, the location of each telescope $i$ is given by the ordered pair $\left(x_{i}, y_{i}\right) \in \mathbb{R}^{2}, i \in\{1, \ldots, N\}$ subject to $x^{L}<x_{i}<x^{H}$ and $y^{L}<y_{i}<y^{H}$.

In the following, we present four important features to describe the overall performance of an array of Cherenkov Telescopes and we use them to conceive the functions $f_{i}$ that will be later implemented in the optimization algorithm as objective functions. These features are effective area, mean trigger multiplicity, angular resolution and energy resolution. It is important to stress that here we are only concerned with presenting the descriptive equations of each performance function, so that more details about the physical aspects involved can be found in [8]. In order to deal computationally with angular and energy resolutions, these features are not calculated exactly. Instead of that, we use heuristic functions, based on averages of some essential components of the angular and energy resolutions.

I) The Effective Area $A_{e f}$ is given by the integral of the probability $P$ of detection of events by all $N$ telescopes over the shower impact area $A$, i.e.,

$$
A_{e f}=\int P \mathrm{~d} A \text {. }
$$

Note that $P$ depends on the positions of all $N$ telescopes, the choice of the minimum telescope trigger multiplicity to validate a stereoscopic detection and 
also the trigger probability function of each telescope. In this work, we take at least three triggering telescopes as the condition for stereoscopic detection.

II) The Mean Trigger Multiplicity $<k>$ is defined as the weighted mean

$$
<k>=\frac{\sum_{k=3}^{N} A_{k} \cdot k}{A_{e f}},
$$

where $k$ corresponds to all possible number of telescopes that can be triggered by an event in a stereoscopic detection, $A_{k}=\int P_{k} \mathrm{~d} A$ and $P_{k}$ are the the probabilities of detection by only $k \in\{3,4, \ldots, N\}$ telescopes and $A_{e f}=A_{3}+A_{4}+\ldots+A_{N}$. A high average trigger multiplicity enhances quality in the identification of primary gammas over the hadronic background and also affects angular and energy resolutions $[16,17,18]$.

III) One definition to angular resolution is the circle around a simulated source containing $68 \%$ of the reconstructed arrival directions [16]. Motivated by [18], we define $\theta_{\mu \nu} \in[0,90]$ as the mean stereo angle of observation of events by any pair of telescopes $\mu$ and $v$, and we put weights depending on Hillas Parameters Size (S) and Length $(1)^{3}$ to define

$$
\Theta=\sin \left(\theta_{\mu v}\right) \cdot\left(S_{\mu}^{-1}+S_{v}^{-1}\right)^{-1} \cdot\left(l_{\mu}^{-1}+l_{v}^{-1}\right)^{-1},
$$

in such a way that the function $\langle\Theta>$ used in place of the angular resolution is then given by the weighted mean of $\Theta$ over the $N$ telescopes and the shower impact area, that is,

$$
<\Theta>=\frac{\int \Theta P \mathrm{~d} A}{\int P \mathrm{~d} A} .
$$

In this way the angular resolution should increase as $\langle\Theta\rangle$ increases.

IV) Energy resolution can be defined as the width of the distribution generated by the relative error between the reconstructed energies and simulated ones from a given source (e.g. [20]). Considered as a function of one telescope depending on the impact parameter of observation ${ }^{4}$, the fluctuations $L$ of the number of photoelectrons detected by a telescope are directly related to the energy resolution (e.g.

\footnotetext{
${ }^{3}$ The reflection in the telescope mirror of the Cherenkov radiation emitted by the shower print an image in the telescope camera in the form of an ellipse whose shape and orientation can be characterized by a set of parameters popularized by Hillas [19]. We are interested in two of these parameters: Size, which is the measure of the total number of photoelectrons recorded by the camera and Length, which is the root mean square of the distribution of the photoelectrons in the camera along the major axis of the ellipse that was fitted to the image.

${ }^{4}$ The impact parameter of observation is the radial distance of a telescope and the center of the shower.
} 
[20]). Hence we take as the function $\langle L\rangle$ the mean value of these fluctuations over the $N$ telescopes and the shower impact area, such that

$$
<L>=\frac{\int L P \mathrm{~d} A}{\int P \mathrm{~d} A} .
$$

Using these four functions and the definition of the constraints in the variable space, we can now write our optimization problem as

$$
\begin{aligned}
& \max \left\{A_{e f},<k>,<\Theta>\right\} \\
& \min <L> \\
& x^{L} \leq x_{i} \leq x^{H} \quad i=1, \ldots, N \\
& y^{L} \leq y_{i} \leq y^{H} \quad i=1, \ldots, N
\end{aligned}
$$

where $\mathbf{v}=\left[\mathbf{x}^{\mathbf{T}} \mathbf{y}^{\mathbf{T}}\right]^{\mathbf{T}} \in \mathbb{R}^{\mathbf{2 N}}$ is a columm vector with the coordinates that determine the position of each telescope on the ground.

\section{The Optimization Algorithm}

This section develops the algorithm created to solve the many-objective optimization problem (7) presented in Section 3. Due to the need to deal with four objective functions, we use the evolutionary algorithm EliteNSGA-III described in $[21,22]$.

In comparison to the standard two-objective algorithm NSGA-II, the main difference is that EliteNSGA-III modifies the way the solutions are selected at each iteration. In comparison to other many-objective evolutionary algorithms, the main features of EliteNSGA-III are the use of reference points inserted a priori in the objective space, whose number, $\mathrm{H}$, is a choice from the Decision Maker and the use of an external population of solutions, denominated elite, whose role is to improve the convergence of the algorithm.

In addition, there are three particular characteristics of the EliteNSGA-III that motivated our choice of using it, namely,

(i) Aptitude to treat more than three objectives, in the context of many-objective optimization;

(ii) Lowest possible dependence on external parameters - only recombination $n_{c}$ and mutation $n_{m}$ indexes were used;

(iii) The maintenance of the Pareto Criterion for non-dominated solutions. 
Next, we present a more detailed description of the algorithm, from its initialization to its stopping criterion, and we conclude the section with a pseudo-code summarizing the main steps of the proposed algorithm.

\subsection{Initialization}

A solution for the array is an individual of $2 N$ real numbers corresponding to the coordinates of the $N$ telescopes. The initial population is generated randomly and uniformly. The population size in the EliteNSGA-III algorithm is chosen to be even, and in such a way that there is at least one solution associated with each reference point. Therefore, the population size is given by the least even number equal to or greater than $H$.

The first elite, with the same size as the initial population, is initialized with the worst values for each objective and the telescope's positions at infinity.

\subsection{Insertion of reference points}

Firstly, the objective functions are normalized in the interval $[0,1]$. Then, some equally spaced reference points are added into the objective space, as shown in Figure 1 for bi and three-dimensional cases. The $H$ number of reference points depends on the spacing between them and the $M$ number of objective functions. Let $p$ be the number of spacings between the reference points along any coordinate axis.

\subsection{Creation of the offspring solutions}

At each iteration, the algorithm works with two generations, $P$ and $Q$. Let $P_{t}$ be the population created by the candidates to solve the problem at iteration $t$ of the algorithm. The descending population $Q_{t}$ is generated from $P_{t}$ by the Simulated Binary Recombination [23] and Polynomial Mutation [13] defined in the Appendix. After that, these two populations compete with each other to generate a new population $P_{t+1}$.

\subsection{Genetic operators}

The recombination occurs among individuals of $P_{t}$ and the elite, where each individual of the population or elite is taken with $50 \%$ probability to participate in it. We set the index $n_{c}=30$.

The probability of a gene in each individual being mutated is taken to be equal to the inverse of the number of variables of $\mathbf{v}$. The mutation operator was used with index $n_{m}=20$.

\subsection{Sorting the solutions and the diversity strategy}

Using the non-dominance criterion, the solutions in $R=P \cup Q$ are ranked in non-dominated fronts, $F_{i}$.

Given an iteration $t$ of the algorithm, the population $P_{t+1}$ will be generated by the non-dominated solutions of the fronts in $R_{t}$, receiving one front at a time. Since 

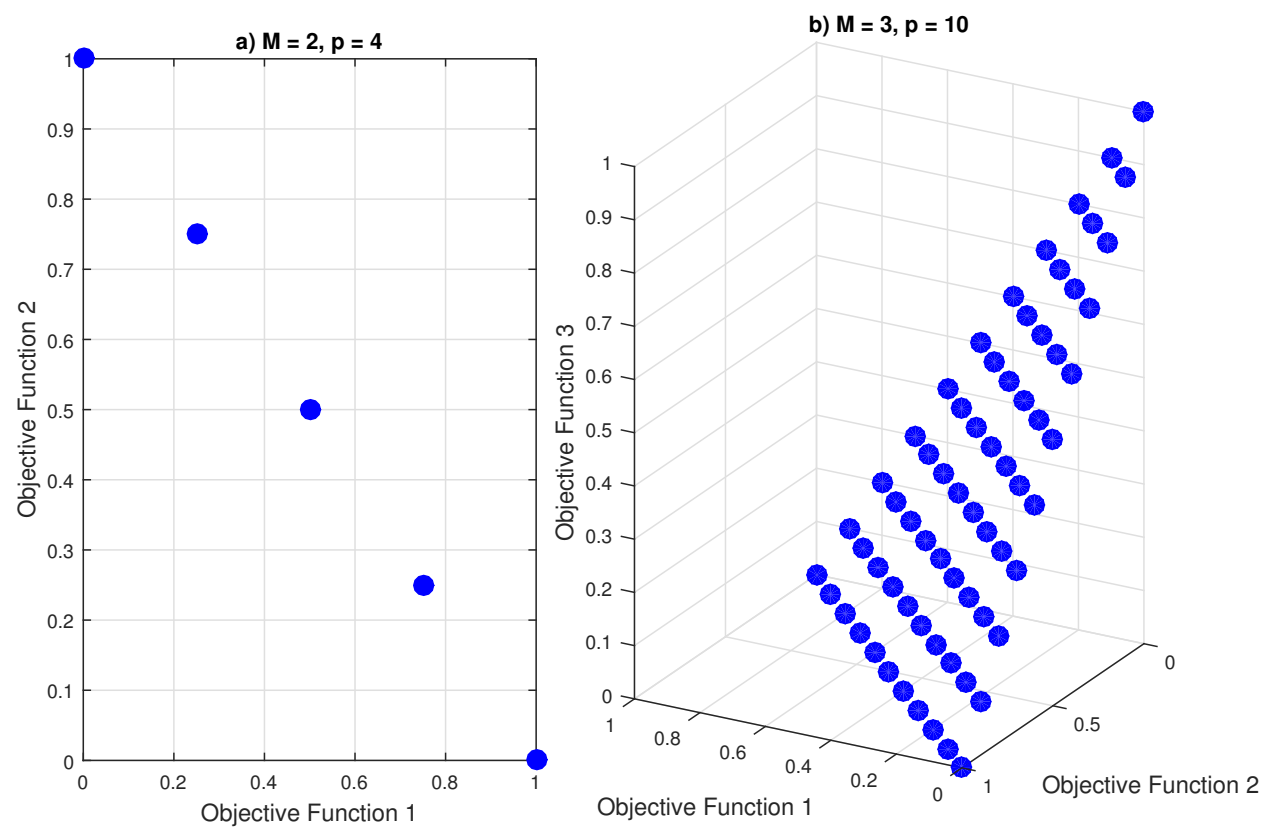

Figure 1: Example of the reference points generated by the NSGA-III algorithm in a problem with a) two or b) three already normalized objectives and with a) four or b) ten spacings between the points.

$P$ has a fixed size equal to $N$ and $P \cup Q$ has $2 N$ solutions, not all fronts will be accommodated. When the last front, $F_{l}$, is admitted, the reference points are used to select which solutions from it will be accommodated. The fronts $\left\{F_{i} \mid i>l\right\}$ will be discarded.

Let $\mathscr{F}=\cup_{i=1}^{l} F_{i}$ be the set that contains the next generation $P_{t+1}$. The next step of the algorithm is to calculate the distance between each individual from $\mathscr{F}$ and each of the $H$ straight lines through the origin and the reference points.

For the elements of $\mathscr{F}$, we find which is the reference direction whose distance from that individual is smallest. Then, we are able to associate a reference point to each individual.

The individuals in the set $\cup_{i=1}^{l-1} F_{i}$ are already accommodated in $P_{t+1}$. For them, we count how many are associated with each reference point. Let $\rho_{j}$ represent the counting of the reference point $j$. On this way we are able to select the remaining individuals from $F_{l}$.

To accomplish this, we randomly select, if it exists, a reference point $\lambda_{j}$ whose count is $\rho_{j}=0$. For all the front individuals $F_{l}$ associated with $\lambda_{j}$, we look for that one - if any - whose distance to the point $\lambda_{j}$ is smallest. That will be chosen to 


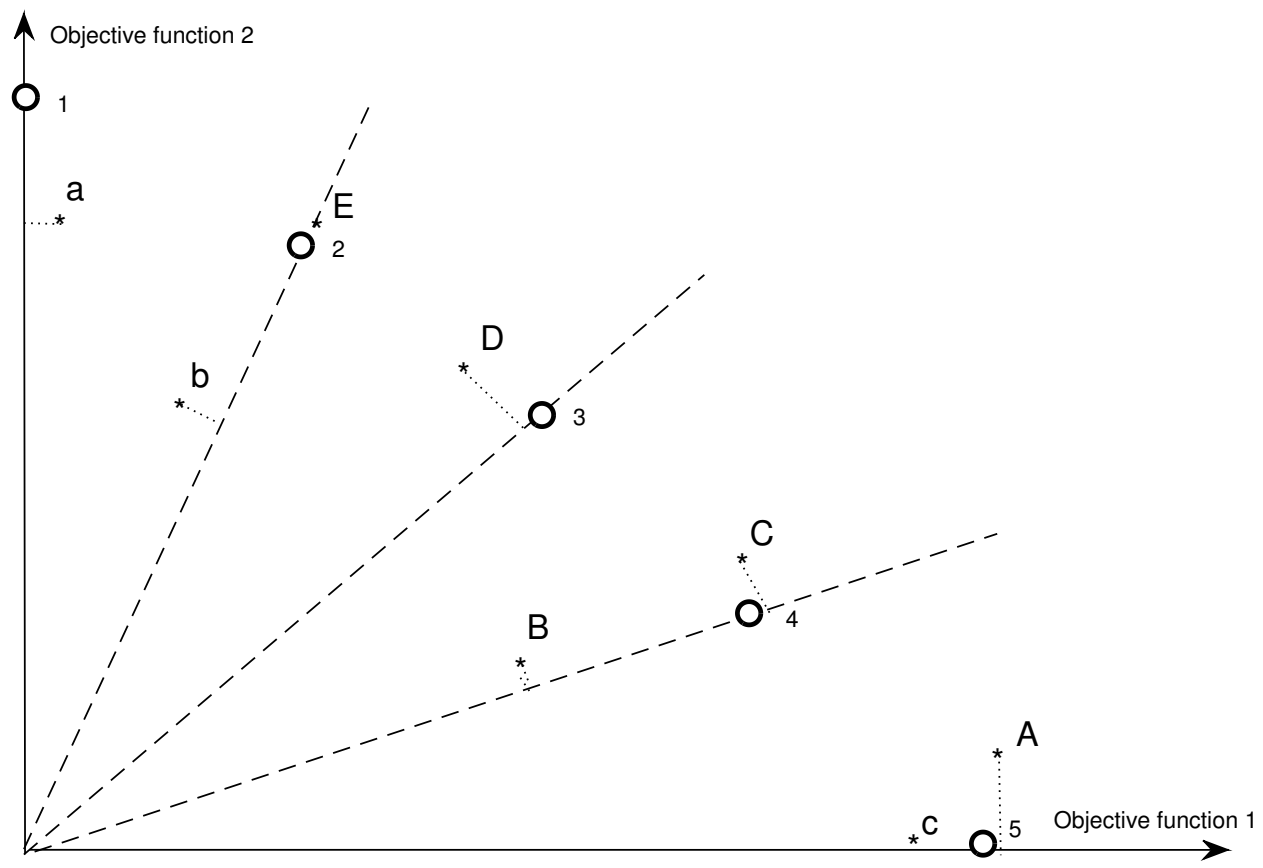

Figure 2: Example showing the diversity strategy based on reference points used in the algorithm. Consider a two-dimensional problem with 5 reference points, $\{1,2,3,4,5\}$. The solutions $\{a, b, c\}$ have already been accommodated in the next generation. Among the remaining 5 solutions, $\{A, B, C, D, E\}$ belonging to the front $F_{l}$, suppose that 3 must be chosen. In this situation, we have that $\rho=(1,1,0,0,1)$. Solutions B and D certainly will be chosen. The latter solution is chosen randomly among the solutions $\mathrm{A}, \mathrm{B}$ and $\mathrm{E}$.

compose the next generation $P_{t+1}$. In this case, we add one to the value of $\rho_{j}$. The procedure is repeated for all reference points such that $\rho_{j}=0$ until $N$ individuals have been selected for the next generation $P_{t+1}$.

If there are no points else such as $\rho_{j}=0$, we move successively to cases $\rho_{j}=$ $1,2, \ldots$ until we have $N$ individuals selected from $F_{l}$. For these cases, however, a random solution of $F_{l}$ that is associated with the point $\lambda_{j}$ will be chosen, and not the one with the shortest distance. The procedure is illustrated in Figure 2.

\subsection{Elite}

Each reference point have at most one member of the elite associated with it. After the population $P_{t+1}$ is generated, for each reference point, we find which of the solutions associated with it has the lowest norm. If it is less than the norm of 
the elite member already associated with that point in the previous generation, then it will become part of the elite, replacing the previous member.

\subsection{Pseudo-code and final remarks}

The algorithm stops after reaching a given number of generations.

The steps of the algorithm explained in Sections 4.1-4.6 are summarized in the pseudo-code in Figure 3.

It is worth emphasizing that differently from the implementation presented in [21], here we adopted the suggestion of [24] for the solutions normalization, in order to reduce the computational cost originally involved in the normalization procedure. Also, the ideal points of each of the $M$ goals were updated at each generation through the populations of the current generation and their offspring, to avoid the need to solve $M$ scalar optimization problems before applying the multiobjective method.

\section{Computational Implementation and Discussion of the Results}

In this section we analyze three different experiments to optimize the IACT array using the objective functions employing the EliteNSGA-III algorithm described in Section 4.

Figure 4 shows the trigger probability functions used to calculate the effective area given in equation 2 .

Table 1 summarizes the cases to be analyzed and the parameters of the algorithm used. 


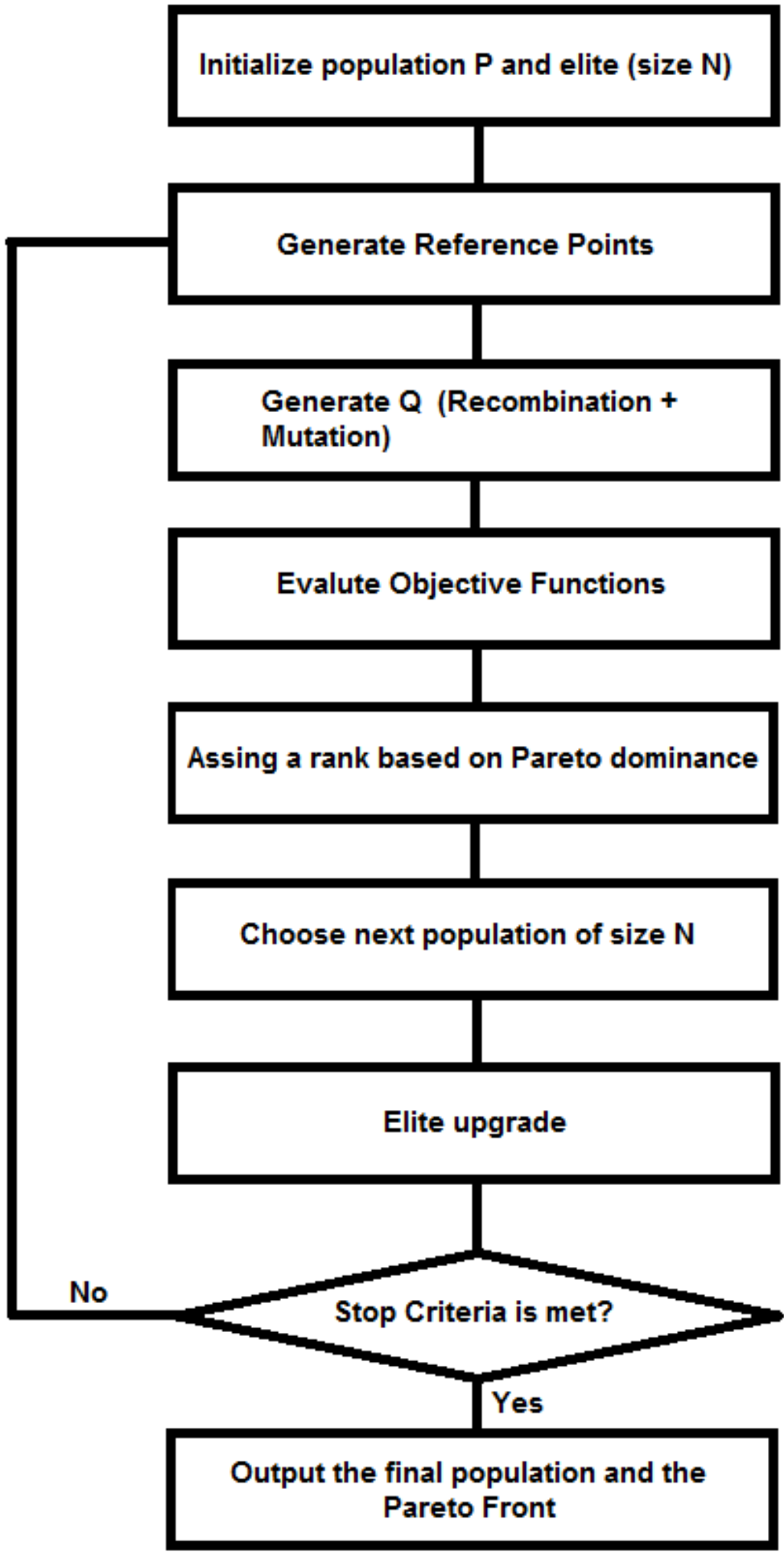

Figure 3: Flowchart of the EliteNSGA-III algorithm. 


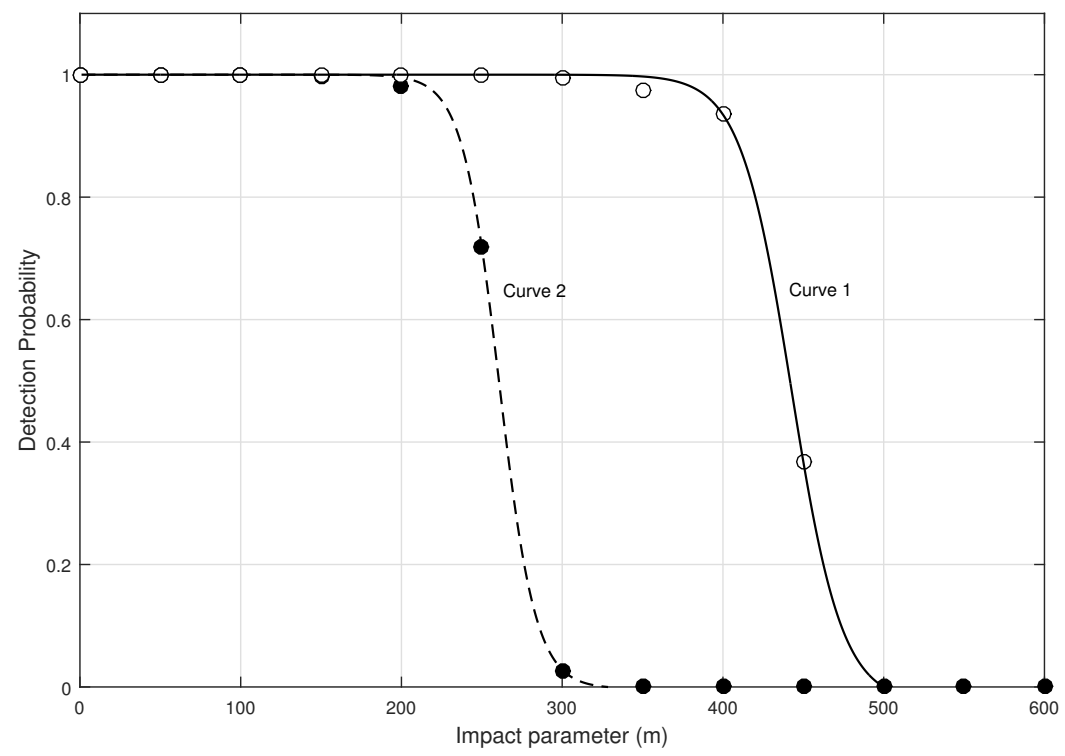

Figure 4: Probability of detection by the telescopes used in this work as a function of the shower impact parameter.

\subsection{First Experiment: Optimization of $A_{e f}$ and $\langle k\rangle$}

This experiment considers only the effective area and the trigger multiplicity to evaluate the performance of the IACT array, in an initial attempt to simplify the analysis and the visualization of the Pareto front. We investigate how effective area in (2) and trigger multiplicity in (3) are related each other, since the former is directly proportional to the detection rate of events and the latter refers to the quality of the reconstruction. Note that (2) and (3) are exact descriptions.

For 3, 4 and 5 telescopes in the array, the solution pointed out by the algorithm places approximately all the telescopes in the same position ${ }^{5}$. This result was expected because the minimum multiplicity of detection was given as equal to three. This can be understood as follows. One shower radiates on the ground focusing most of its radiation in a circular region, with radius approximately 120 $\mathrm{m}$. Hence, when the telescopes are arranged within this area, they are able to detect the light emitted by this shower. In the general case, for showers with different

\footnotetext{
${ }^{5}$ More precisely, the solution places the telescopes with spacing about $20 \mathrm{~m}$ among them, which in fact is the order of magnitude of the length of a telescope.
} 
Table 1: Parameters of the algorithm in each of the cases analyzed. It is shown the number of telescopes in the array, the population size, the dimensions of the area where the telescopes are settled, the recombination and mutation indexes and which detection probability function from Figure 4 was used.

\begin{tabular}{|c|c|c|c|c|c|c|}
\cline { 2 - 7 } & $\begin{array}{c}\text { Number } \\
\text { of } \\
\text { Telescopes }\end{array}$ & $\begin{array}{c}\text { Population } \\
\text { Size }\end{array}$ & $\begin{array}{c}\text { Array } \\
\text { Dimensions }\end{array}$ & $n_{c}$ & $n_{m}$ & $\begin{array}{c}\text { Detection } \\
\text { Curve }\end{array}$ \\
\hline \multirow{3}{*}{$\begin{array}{c}\text { First } \\
\text { Experiment }\end{array}$} & 3 & 22 & $2200 \mathrm{~m} \times 2200 \mathrm{~m}$ & 2 & 10 & 1 \\
\cline { 2 - 7 } & 4 & 22 & $2200 \mathrm{~m} \times 2200 \mathrm{~m}$ & 30 & 20 & 1 \\
\cline { 2 - 7 } & 5 & 22 & $2200 \mathrm{~m} \times 2200 \mathrm{~m}$ & 30 & 20 & 1 \\
\hline \multirow{3}{*}{$\begin{array}{c}\text { Second } \\
\text { Experiment }\end{array}$} & 4 & 44 & $1000 \mathrm{~m} \times 1000 \mathrm{~m}$ & 30 & 20 & 1 \\
\cline { 2 - 8 } & 4 & 22 & $2200 \mathrm{~m} \times 2200 \mathrm{~m}$ & 30 & 20 & 1 \\
\hline $\begin{array}{c}\text { Third } \\
\text { Experiment }\end{array}$ & 6 & 22 & $2200 \mathrm{~m} \times 200 \mathrm{~m}$ & 30 & 20 & 1 \\
\hline
\end{tabular}

impact points, Figure 5 (left column) shows the probability of detection in each of these points and how it decreases if the separation between the telescopes increases. Thus, the array configuration with the telescopes in the same position enhances the detection of showers with different points of impact.

Figure 6 exemplifies how the two objective functions for the entire population converge over 50 generations. The cases with 3 and 5 telescopes generate similar figures indicating the convergence of the Pareto front to a single point, which means the two objectives in these cases are not in conflict.

For 6 telescopes, the array with greater effective area is two disjoint groups with three telescopes each, as Figure 7 shows. The other geometries of the Pareto front are also two groups with three telescopes each, which differ from each other by the separation between the groups. As the average trigger multiplicity increases, these two groups become successively closer. In this case, the objective functions conflict with each other.

This occurs because the minimum multiplicity of detection was chosen as three so, to increase the effective area, it is more advantageous to separate the six telescopes into two groups of three instead of attaching the six telescopes in close positions. For more than 6 telescopes, the array with greater effective area consists in disjoint groups with three telescopes each.

The obtained geometries with the telescopes placed approximately in the same 

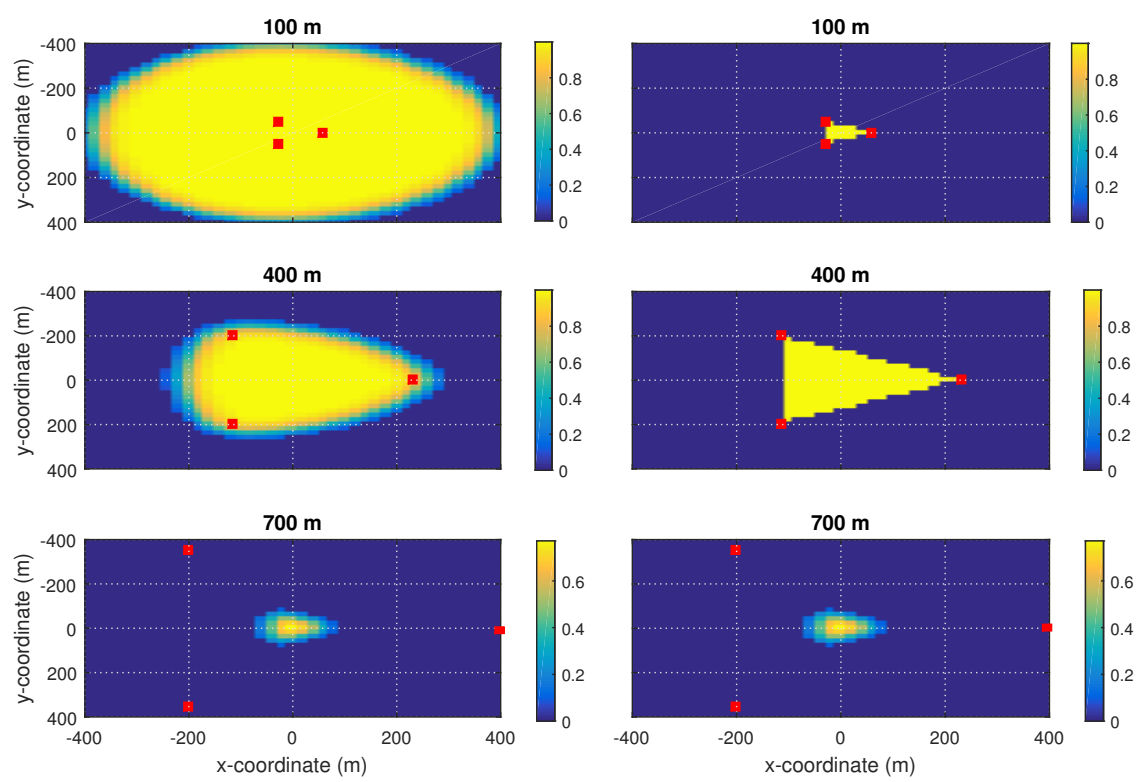

Figure 5: Left: Example of detection probability by three telescopes values at each point on the shower impact area. Different spacing between the telescopes are illustrated: $100 \mathrm{~m}, 400 \mathrm{~m}$ and $700 \mathrm{~m}$. Right: Detection probability values only at the internal area of the array.

position, for example, those of Figure 7, or with aligned telescopes are degenerated solutions: they are not interesting, because these geometries degrade the stereoscopic view of the shower and therefore also the reconstruction of the primary gamma ray [25]. This means we must include other functions to the optimization problem. One way to accomplish this is to optimize the internal area of the array and the average trigger multiplicity there, because the internal area is considered to have better shower reconstruction properties.

\subsection{Second Experiment: Optimization of internal $A_{e f}$ and internal $<k>$}

We define the internal area of some configuration as follows. Given $N$ points in a plane representing the telescopes, straight lines are constructed between all pairs of points, so that the array internal area is the largest convex polygon that can be created with these lines. Figure 5 (right column) illustrates the internal area for the case with 3 telescopes. Also, Figure 5 shows how the separation of the telescopes increases the internal area. 


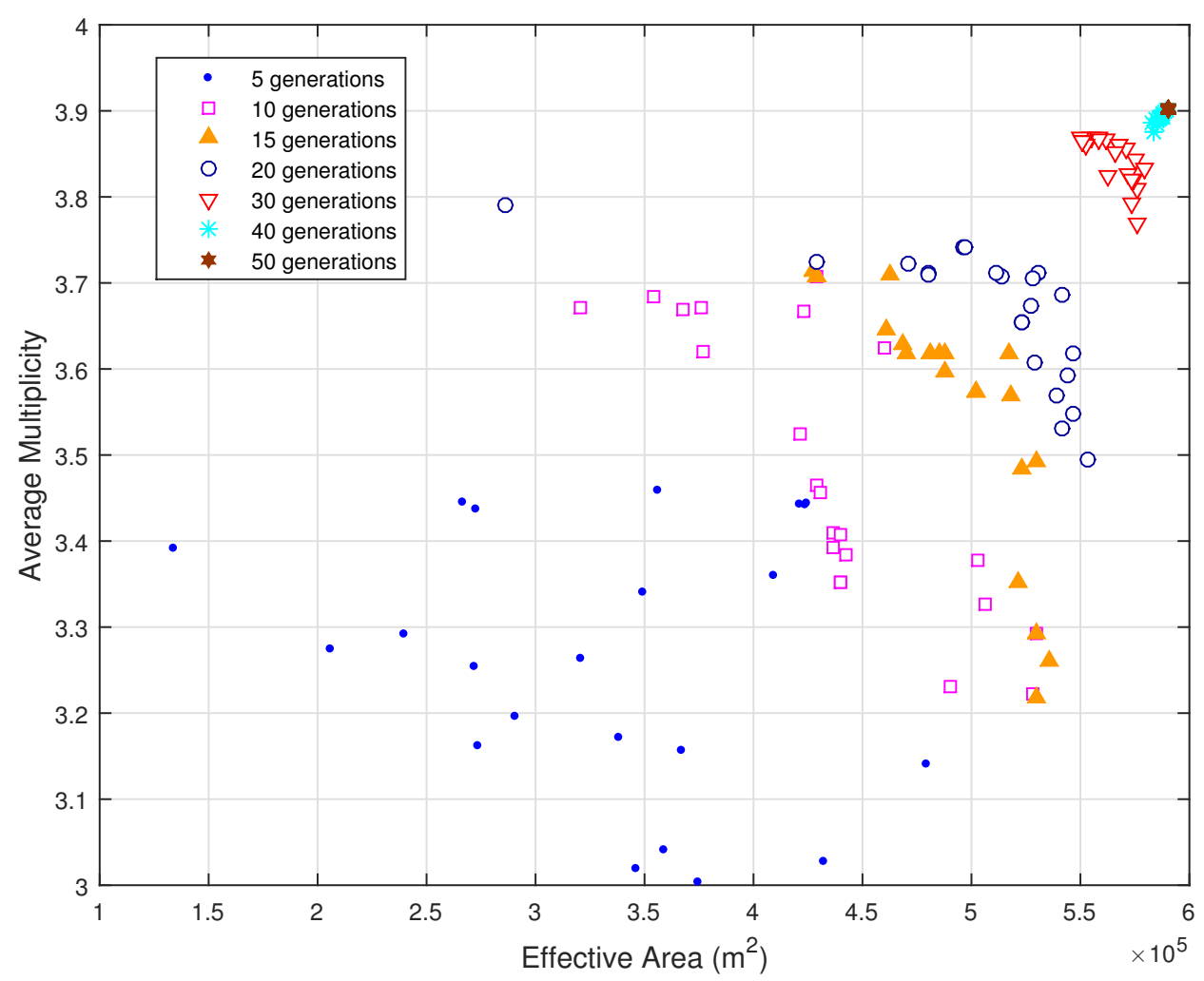

Figure 6: Effective Area and Trigger Multiplicity as a function of the number of generations for 4 telescopes. The points in the Figure show the objective values for the whole population. The converging geometry are the four telescopes in close positions, tending to the same point.

Note that the internal area reaches a maximum value when the separation of the telescopes is equal to the their range of detection. Therefore, it is expected that replacing $A_{e f}$ by the internal $A_{e f}$, the algorithm will find different geometries to avoid the tendency of joining the telescopes into small sub-arrays with three telescopes each.

Here we run again the algorithm in the four situations of Section 5.1, maintaining the number of telescopes but calculating the effective area and the mean trigger multiplicity only in the internal area of the array.

For 3 telescopes, we found approximately regular triangular geometries with sides about $500 \mathrm{~m}$. In fact, this spacing is the scale of the range of detection of the telescopes, as shown in Figure 4 . In the case of 4 telescopes, the solutions found are approximate rectangles with sides ranging from $250 \mathrm{~m}$ to $350 \mathrm{~m}$. 


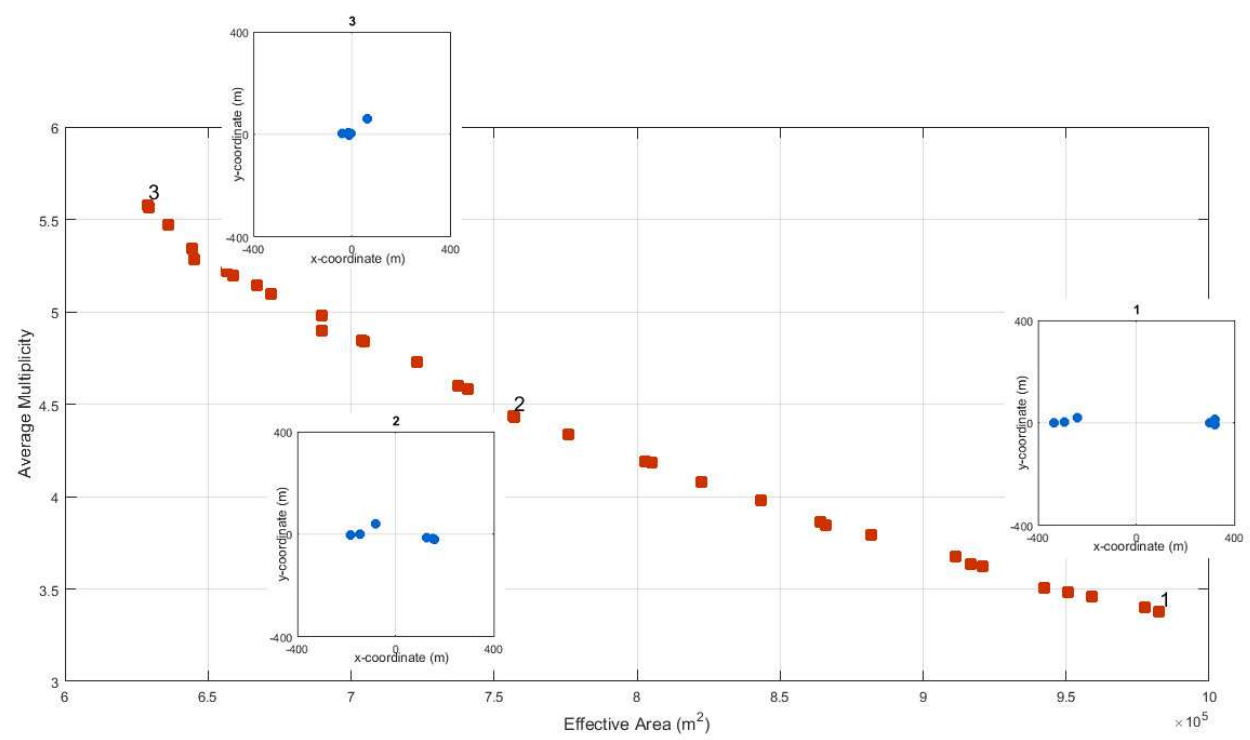

Figure 7: Effective area and average trigger multiplicity for six telescopes. The geometries are composed by subgroups with three telescopes each which differ by the spacing between them. The geometries of three particular solutions are shown.

For 5 and 6 telescopes, more than one geometry is found along the Pareto front. Figure 8 and Figure 9 show the results for 5 telescopes. We have that $60 \%$ of the solutions are face-centered squares and they are the solutions with greater effective area. The other solutions are contained in the Pareto front region with the highest multiplicities. Figure 10 shows the trend for the geometry obtained by inspection of the geometries at Figure 9.

Figure 11 and Figure 12 show the solution for 6 telescopes. Also, about $60 \%$ of the solutions are pentagons with a telescope in the center. These cases point out that not just the scale of separation among the telescopes decreases as the trigger multiplicity increases, but we find that the geometry of the array itself can also change. This is an example of the kind of the optimization result that can be obtained by the algorithm proposed here. Figure 13 shows the trend for the geometry obtained by inspection of the geometries at Figure 12.

We conclude this section remarking that the optimization of the internal effective area and internal average trigger multiplicity indeed avoids the degenerated solutions. In spite of the internal effective area being one of the parameters used to optimize cell arrays, the whole area of observation must be included, because the 


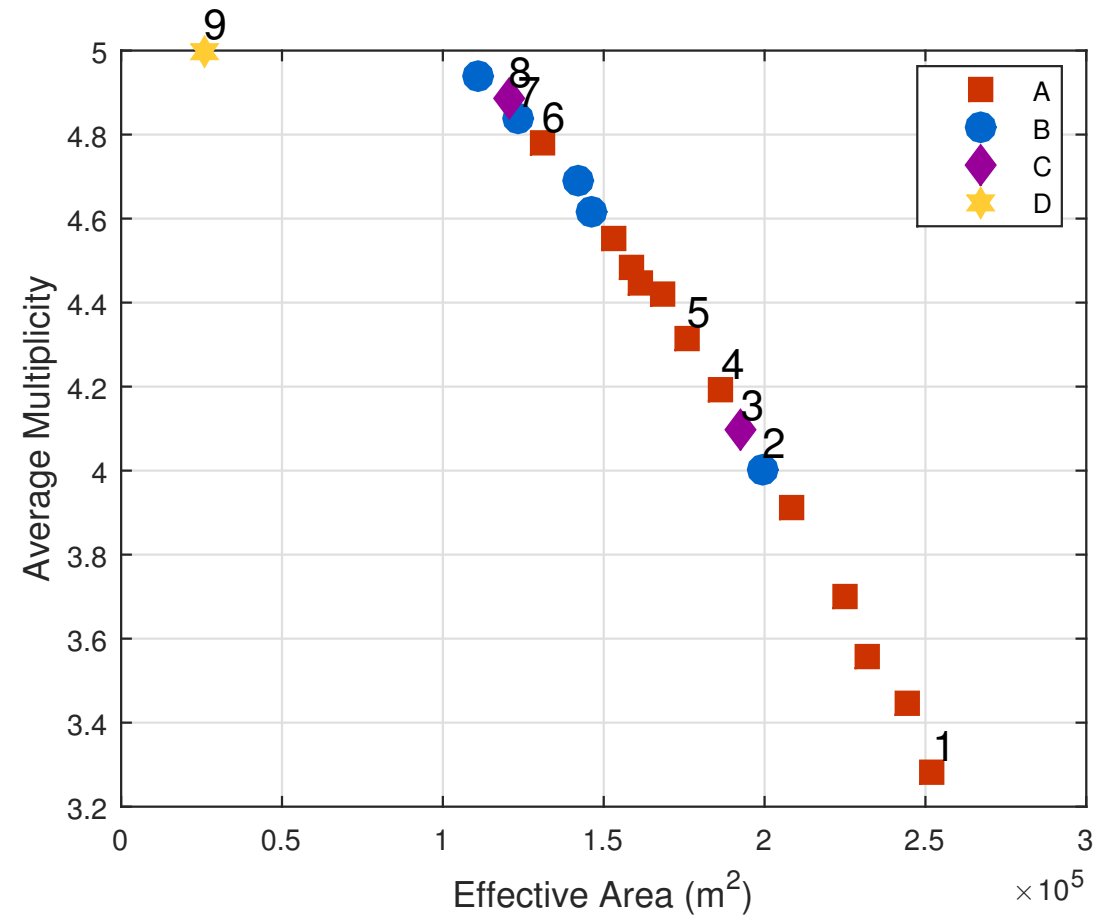

Figure 8: Pareto Front for the effective internal area and average trigger multiplicity for 5 telescopes. Similar geometries were grouped by capital letters and they are shown in Figure 9.

external areas can play an important role in the optimization of the array. Hence, in the next section, we optimize an array using all the four objective functions of Section 3 .

\subsection{Third Experiment: Optimization using the four objective functions}

In this section, we deal with the full many-objective optimization problem (7) to find configurations to an array with four telescopes. Figure 14 shows the Pareto Front obtained and Figure 15 highlights the geometries found.

Figure 14 shows that the Pareto front approaches a plane in the objective space. In order to compare the output of the algorithm with an intuitive solution given by a square array with sides equal to $100 \mathrm{~m}$, we included this solution in Figure 14 and denominated it by $Q_{100}$. The ideal solution ${ }^{6}$ was included too.

\footnotetext{
${ }^{6}$ The ideal solution is the point in the objective space obtained by optimizing the objective functions separately. Since these functions are in conflict, the ideal solution is non-physical.
} 

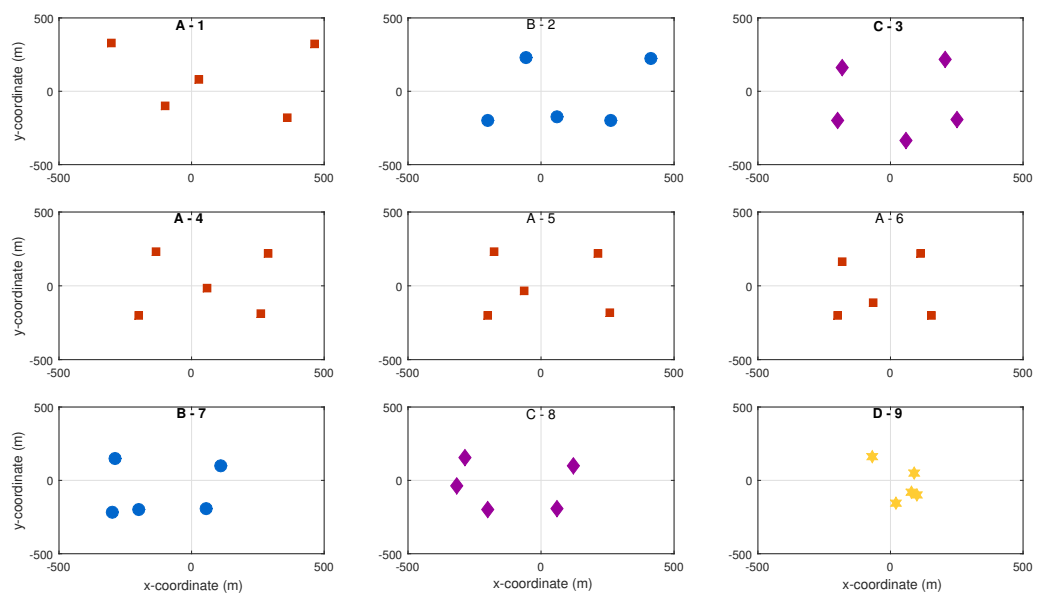

Figure 9: The four typical geometries for five telescopes, showing in particular the nine solutions numbered in Figure 8.

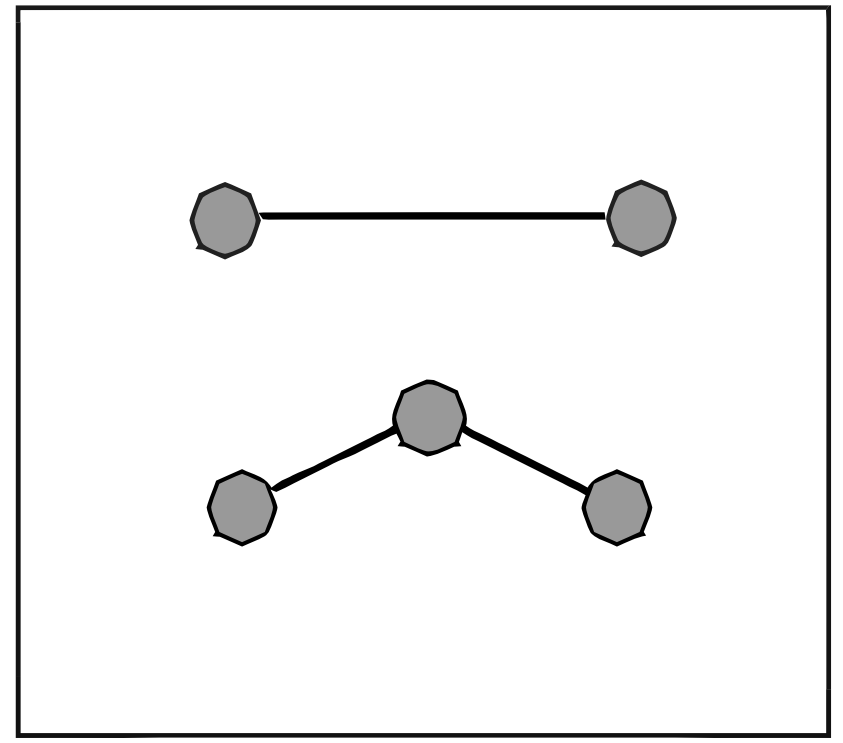

Figure 10: Conclusion for the case of five telescopes, showing an intermediate geometry between the pentagon and the array composed by two parallel lines.

Figure 14 shows that array " 2 " can be a better alternative to the array $Q_{100}$. Both have the same effective area, but array "2" has a higher value for the objec- 


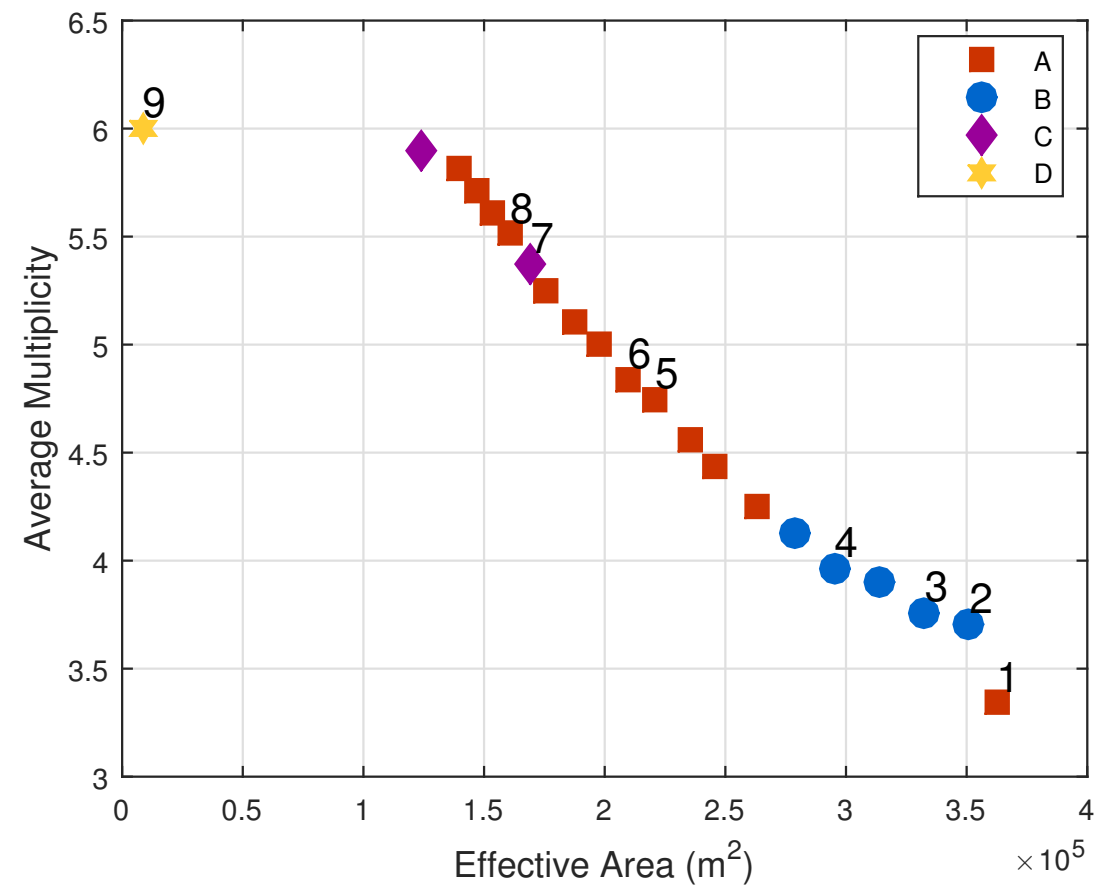

Figure 11: Pareto Front for the effective internal area and average trigger multiplicity for 6 telescopes. Similar geometries were grouped by capital letters and they are shown in Figure 12.

tive function $\langle\Theta\rangle$. It consists of a triangle with sides measuring $L$ and with a telescope in its center.

Thus, given the appearance of this unconventional geometry, we perform a last experiment to compare it with two traditional four-sized polygons: squares and diamonds. We fixed squares and diamonds arrays of different spacings and calculated the four objective functions for them. Figure 16 shows the results in the objective space, comparing them to the face centered triangles geometry. We conclude that for this geometry, the arrays achieve better results for the four objectives. Note that for spacings greater than $\sim 350 \mathrm{~m}$, this behavior is not verified, but they consist of solutions with effective area decreased by an order of magnitude.

This experiment illustrates the methodology employed to obtain new geometries. In order to be accepted, the face centered triangles geometry must necessarily be tested by the detailed Monte Carlo simulation of the array. 

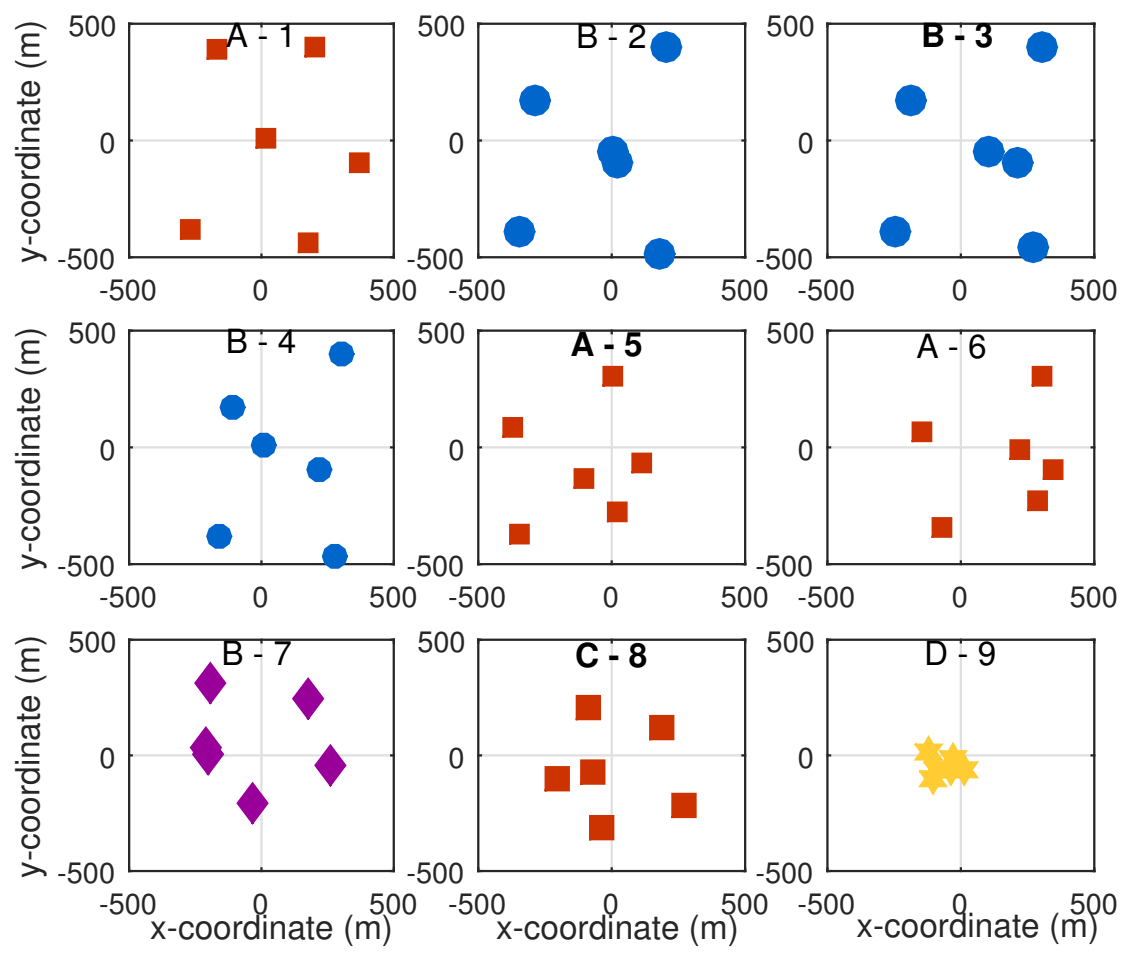

Figure 12: Geometries for the solutions numbered in Figure 11.

\section{Conclusions}

In this paper, we have proposed the use of an evolutionary algorithm as a tool to guide the search for an optimal configuration of an Imaging Atmospheric Cherenkov Telescopes array. We have done three simple experiments to illustrate the methodology.

As the number of telescopes in the array increases, it is more advantageous to increase the effective area by separating the telescopes rather than improving the detection at the edges of the array. For a detection criterion that considers minimum multiplicity equal to three, this occurs for arrays with six or more telescopes. The average trigger multiplicity, on the other hand, decreases if the separation among the telescopes is greater. For example, for nine telescopes with a minimum multiplicity of three, the configuration with the largest effective area and the smallest multiplicity consists of three clusters with three telescopes each. In fact, as the multiplicity increases, not only does the separation scale among telescopes decrease, but we found that the geometry itself of the array can also change.

An interesting result is the region in the search space of an array with four tele- 


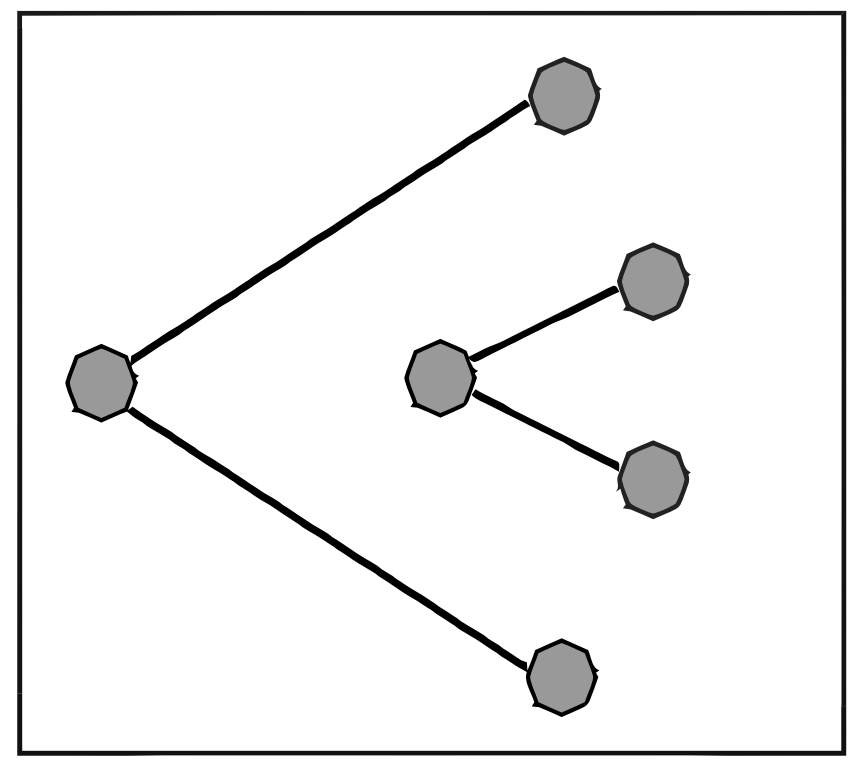

Figure 13: Conclusion of the case of six telescopes, showing a geometry composed of two triangles.

scopes with better values for the objective functions. This region is all composed by face-centered triangular geometries and it was obtained by an evolutionary algorithm. The next step would be to compare this result with the square and diamonds geometries employing the detailed Monte Carlo simulation of the array.

In order to understand the conflict among the objective functions and their relation with the geometry of the array, we were able to create for 5 and 6 six telescopes a partition of the Pareto front in optimal geometries according to the optimization objective. Indeed, the importance of understanding the conflict among the objective functions comes from the astrophysical motivations for the experiment. In [20], the compromise between resolution in energy and effective area is highlighted and they choose the detection area as the priority for high energy events, which have a low rate. For cosmological sources, the priority is not to study the morphological characteristics of distant sources with an optimum angular resolution. Finally, if any source has a cut in the spectrum, it is of interest to know as accurately as possible the energy in which the cut occurs, so that the resolution in energy is the priority in this case.

Since the problem is many-objective, the set of geometries obtained by the evolutionary algorithm has a great diversity, which causes difficulty for the decision 


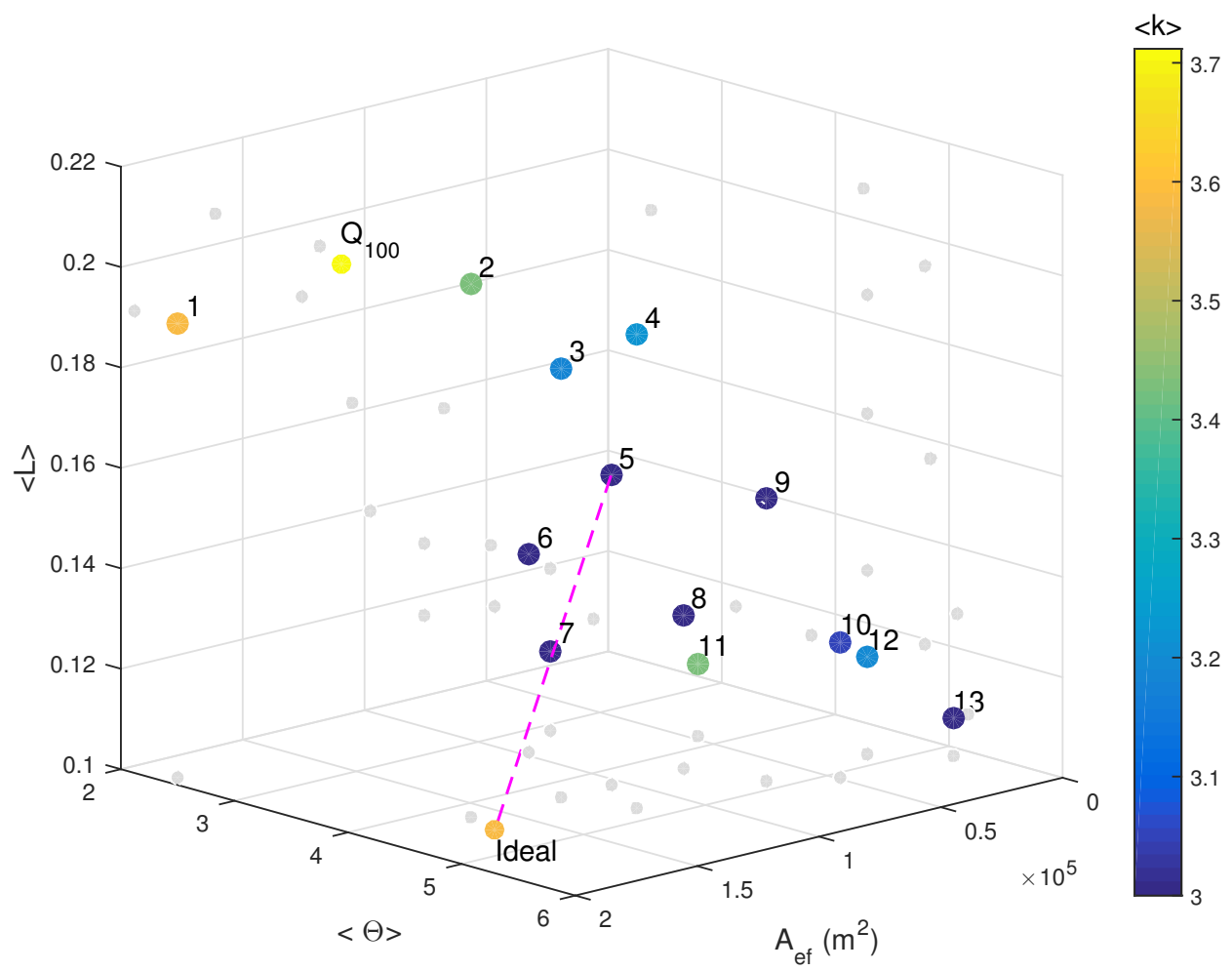

Figure 14: Pareto front in the case of four telescopes. The $\langle L\rangle$ function is minimized and the others are maximized. Array "5" is the solution closer to the ideal point in the normalized three-dimensional space. $Q_{100}$ corresponds to four telescopes arranged in a square of side $100 \mathrm{~m}$.

maker to choose one solution, but this diversity may include the desired solutions to the problem and should be followed by Monte Carlo investigation. In fact, the diversity of solutions can decrease if we employ more accurate and restrictive models.

We emphasize that dealing with many-objective problems, usually more than four objectives, is still an open problem [26]. This case has some peculiarities and there is no consensus today as to what are the best algorithms to treat it, if they exist.

Finally we list some future applications of the tools developed here. The most general application of the evolutionary algorithm would be to search both the geometry of the array and the telescopes parameters.

More generally, we would like to know if the algorithm could also be useful in 

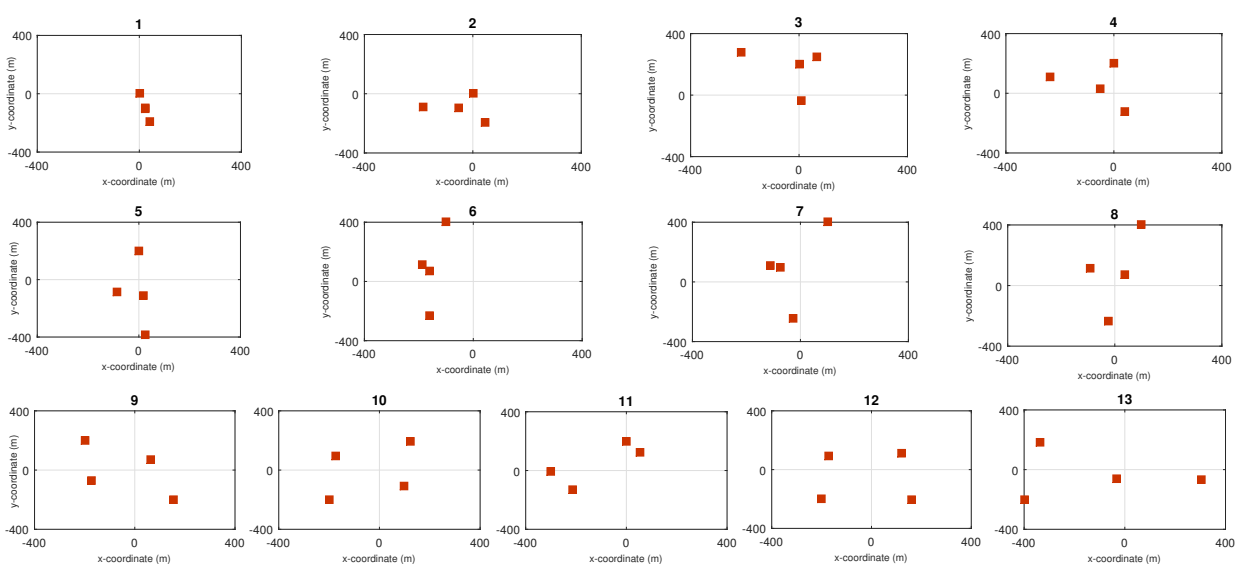

Figure 15: Arrays on the Pareto front according to the indexes of Figure 14.

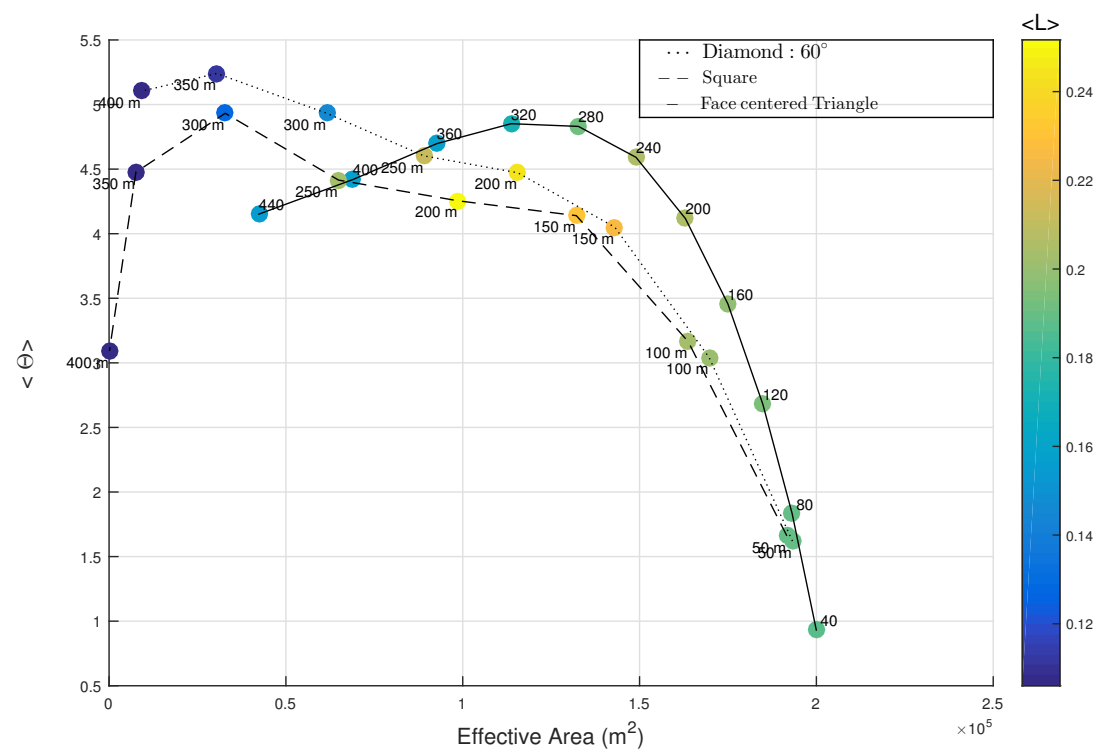

Figure 16: Values of $A_{e f},\langle\Theta>$ (to be maximized), and $\langle L\rangle$ (to be minimized), calculated for arrays with different scales. The geometries compared are the face centered triangles - as a result from the evolutionary algorithm -, and diamonds with different values for the aperture angle: $90^{\circ}$ (lines) and $60^{\circ}$ - as the results from simulations. 
other applications for the Cherenkov Telescope Array. For example, the cost for constructing an array can be given as a constraint on the problem or it can be given as an optimization goal. Other example could be the employment of the algorithm to help finding a geometry for some possible expansion of an already constructed array.

The Pareto fronts obtained by this method are ways of helping the experimenter to decide the array to be built. More generally, for an array already built with many telescopes, the results could help in deciding which telescopes to use to perform certain observation with given scientific objectives.

Acknowledgments. We thank the Brazilian National Council for Scientific and Technological Development for financial support.

\section{Appendix}

This appendix defines the Recombination and Mutation operators used in Section 4.4 .

An individual in the population is represented by a number of real variables. Let $a$ and $b$ be two variables that belong respectively to two individuals that were selected by recombination, where $b>a$. The Simulated Binary Recombination [23] operator generates two new variables $x_{1}$ and $x_{2}$ through the equation:

$$
\begin{aligned}
& x_{1}=0,5(a+b-\beta|b-a|) \\
& x_{2}=0,5(a+b+\beta|b-a|),
\end{aligned}
$$

where

$$
\begin{aligned}
\beta & =(u \alpha)^{\frac{1}{n_{c}+1}}, & & u \leq 0,5 \\
& =\left(\frac{1}{2-u \alpha}\right)^{\frac{1}{n_{c}+1}}, & & u>0,5
\end{aligned}
$$

and $u$ is a random number in the interval $[0,1], \alpha=2-\Delta^{-\left(n_{c}+1\right)}$ and

$$
\Delta=1+\frac{2 \min \left(a-x_{\min }, x_{\max }-b\right)}{b-a},
$$

in which $x_{\min }$ and $x_{\max }$ are the are the lower and upper bounds of the interval that contains the problem variables. If the domain is unlimited, make $\alpha=2$ in the equation 10. If $a=b$, we will assume that the two individuals generated are equal to the pair that originated them. We have that $n_{c}$ is an external parameter. Figure 17 shows the effect of varying this parameter. 
Given a probability $p$ of mutation, a random number $u$ is generated in the interval $[0,1]$ for each variable of each individual in the population. If $u<p$, then the mutation operator changes that variable. In general, we adopt $p=1 / N$, where $N$ is the size of the population. Thus, on average one variable is mutated in each individual. Using the Polynomial Mutation [13], if $x \in[a, b]$ is a mutating variable, then the new variable will be given by $x^{\prime}=x+\delta(x-a)$, where $\delta=2 u\left(1+n_{m}\right)-1$, if $u \leq 0.5$; or $x^{\prime}=x+\delta(b-x)$, where $\delta=1-[2(1-u)]\left(1+n_{m}\right)-1,>0.5$. It is assumed that $n_{m}$ is an external parameter, usually $n_{m} \in[20,100]$. Figure 18 shows the effect of the different choices for the $n_{m}$ parameter.

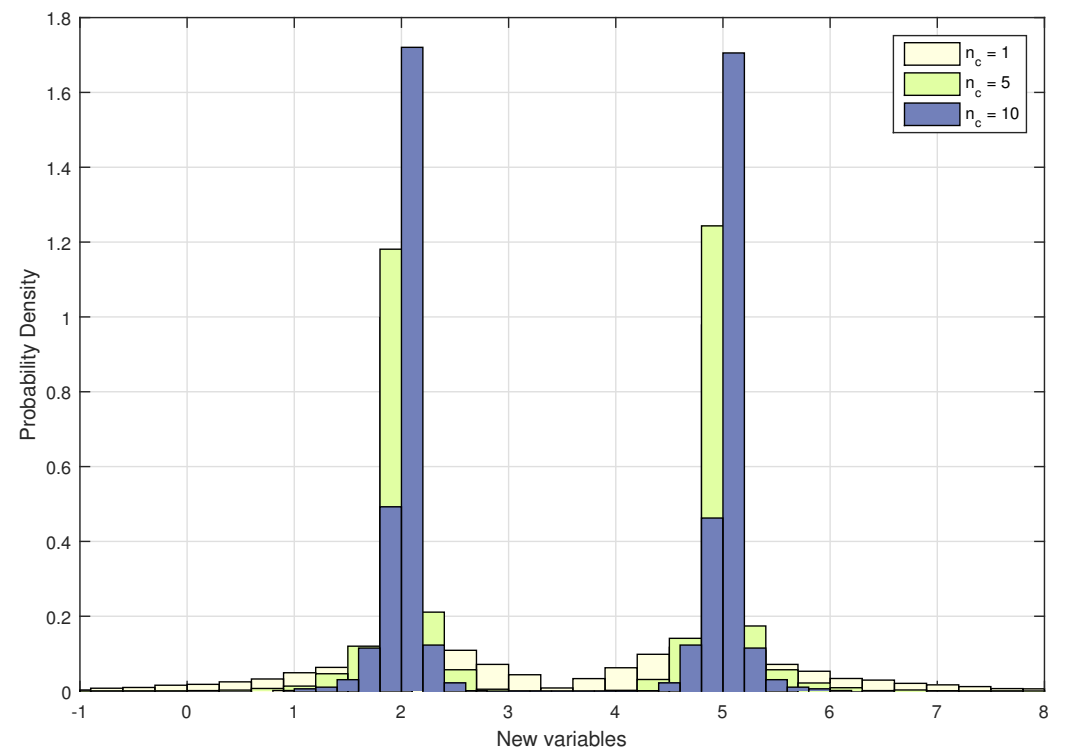

Figure 17: Probability distribution for the variables $x_{1}$ and $x_{2}$ generated from the Simulated Binary Recombination in a one-dimensional example, where variables 2 and 5 participate in the recombination. The distributions have peaks in the two variables that participated in the recombination and in Figure the allowed interval for the new solutions generated is $[-1,8]$. 


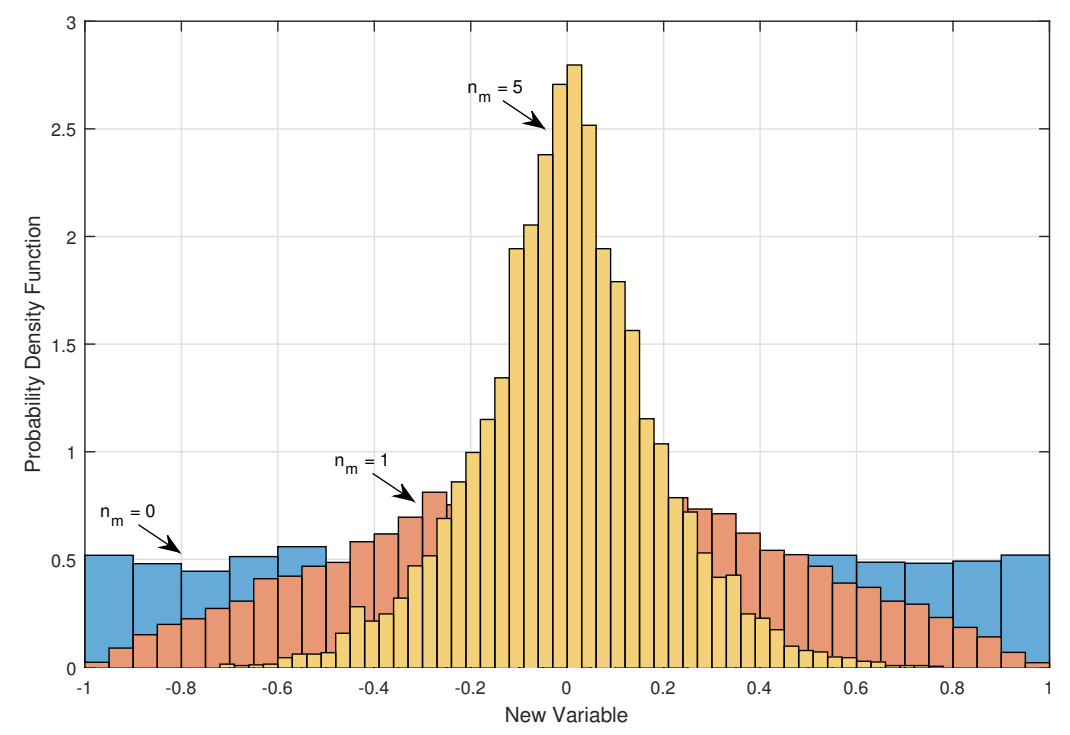

Figure 18: Variable $x^{\prime}$ generated from the Polynomial Mutation acting on a solution $x=0$ in the range $[-1,1]$ for different values of the index $n_{m}$. For $n_{m}=1$, the probability distribution for the mutation is uniform. The distribution becomes narrower as the value of $n_{m}$ increases to the limit $n_{m} \rightarrow \infty$, when the distribution becomes a Dirac Delta so that $x^{\prime}=x$, situation equivalent to there is no mutation.

\section{References}

[1] HILLAS, A. Evolution of ground-based gamma-ray astronomy from the early days to the Cherenkov Telescope Arrays. Astroparticle Physics, Elsevier, v. 43,p. 19, 2013.

[2] WEEKES, T. C. et al. Observation of TeV gamma rays from the Crab Nebula using the atmospheric Cerenkov imaging technique. The Astrophysical Journal, v. 342, p. 379, 1989.

[3] HINTON, J. Ground-based gamma-ray astronomy with Cherenkov telescopes. New Journal of Physics, IOP Publishing, v. 11, n. 5, p. 055005, 2009.

[4] HOFMANN,W.; et al. On the optimum spacing of stereoscopic imaging atmospheric Cherenkov telescopes. Astroparticle Physics, Elsevier, v. 13, n. 4, p. $253258,2000$. 
[5] DENMAN, J. Design studies for a multi-TeV telescope array: PeX (PeV eXplorer). PhD Thesis University of Adelaide, Australia, 2012.

[6] THE CTA CONSORTIUM. Cherenkov Telescope Array: The next generation gamma-ray observatory. ArXiv preprint arXiv:1709.05434, 2017.

[7] HECK, and DIETER, et al. CORSIKA: A Monte Carlo code to simulate extensive air showers. FZKA 6019. Technical Report, 1998.

[8] BERNLHR, K.; et al. Monte Carlo design studies for the Cherenkov Telescope Array. Astroparticle Physics, Elsevier, v. 43, p. 171, 2013.

[9] BERNLHR, K.; et al. Progress in Monte Carlo design and optimization of the Cherenkov Telescope Array. ArXiv preprint arXiv:1307.2773, 2013.

[10] BARNACKA, A.; et al. Performance of the Cherenkov Telescope Array at energies above $10 \mathrm{TeV}$. ArXiv preprint arXiv:1307.3409, 2013.

[11] AHARONIAN, F.; HOFMANN, W.; KONOPELKO, A.; VLK, H. The potential of ground based arrays of imaging atmospheric Cherenkov telescopes I. Determination of shower parameters. Astroparticle Physics, Elsevier, v. 6, n. 3-4, p. 343, 1997.

[12] PANDURO, Marco A. et al. A multi-objective approach in the linear antenna array design. AEU-International Journal of Electronics and Communications, v. 59, n. 4, p. 205, 2005.

[13] DEB, K. Multi-objective optimization using evolutionary algorithms, John Wiley \& Sons, 2001.

[14] Simon, D. Evolutionary Optimization Algorithms, 2013, John Wiley \& Sons.

[15] COELLO, C. A. C. et al. Evolutionary algorithms for solving multi-objective problems, Springer, 2007

[16] MAIER, G.; ARRABITO, L.; BERNLHR, K.; BREGEON, J.; CUMANI, P.; HASSAN, T.; MORALEJO, A. Performance of the Cherenkov Telescope Array. ArXiv preprint arXiv:1709.01381, 2017.

[17] KONOPELKO, A.; et al. Performance of the stereoscopic system of the HEGRA imaging air Cerenkov telescopes: Monte Carlo simulations and observations. Astroparticle Physics, Elsevier, v. 10, n. 4, p. 275, 1999.

[18] BERNLHR, K.; et al. Monte Carlo design studies for the Cherenkov Telescope Array. Astroparticle Physics, Elsevier, v. 43, p. 171, 2013. 
[19] HILLAS, A. M. Cerenkov light images of EAS produced by primary gamma. In: International Cosmic Ray Conference. 1985. v. 3.

[20] ALEKSIC, J.; et al. The major upgrade of the MAGIC telescopes, part II: A performance study using observations of the Crab Nebula. Astroparticle Physics, Elsevier, v. 72, p. 76, 2016.

[21] DEB, K.; JAIN, H. An evolutionary many-objective optimization algorithm using reference-point based nondominated sorting approach, part I: Solving problems with box constraints. IEEE Trans. Evolutionary Computation, v. 18, n. 4, p. 577, 2014.

[22] IBRAHIM, A.; RAHNAMAYAN, S.; MARTIN, M. V.; DEB, K. EliteNSGAIII: An improved evolutionary many-objective optimization algorithm. In: IEEE. Evolutionary Computation (CEC), 2016 IEEE Congress on., 2016. p. 973.

[23] AGRAWAL, R. B.; DEB, K.; AGRAWAL, R. Simulated binary crossover for continuous search space. Complex systems, v. 9, n. 2, p. 115, 1995.

[24] YUAN, Y.; XU, H.;WANG, B. An improved NSGA-III procedure for evolutionary many-objective optimization. In: ACM. Proceedings of the 2014 Annual Conference on Genetic and Evolutionary Computation, 2014. p. 661668.

[25] HOFMANN, W.; JUNG, I.; KONOPELKO, A.; KRAWCZYNSKI, H.; LAMPEITL, H.; PHLHOFER, G. Comparison of techniques to reconstruct VHE gamma-ray showers from multiple stereoscopic Cherenkov images. Astroparticle Physics, Elsevier, v. 12, n. 3, p. 135, 1999.

[26] DUTTA, J.; GUPTA, A.; SENGUPTA, R. N. Decision sciences: theory and practice. CRC Press, 2017.

doi:10.6062/jcis.2018.09.03.0153

(C) Author(s) 2019. This work is distributed under

the Creative Commons Attribution 4.0 License.

(c) 9 